\title{
Programme coverage, condom use and STI treatment among FSWs in a large-scale HIV prevention programme: results from cross-sectional surveys in 22 districts in southern India
}

\author{
Lakshmi Ramakrishnan, ${ }^{1}$ Abhishek Gautam, ${ }^{1}$ Prabuddhagopal Goswami, ${ }_{1}^{1}$ \\ Srinivasan Kallam, ${ }^{1}$ Rajatashuvra Adhikary, ${ }^{1}$ Mandar K Mainkar, ${ }^{2}$ \\ Banadakoppa M Ramesh, ${ }^{3,4}$ Guy Morineau, ${ }^{5}$ Bitra George, ${ }^{1}$ Ramesh S Paranjape ${ }^{3,4}$
}

${ }^{1}$ Family Health International, New Delhi, India ${ }^{2}$ National AIDS Research Institute, ICMR, Pune, India ${ }^{3}$ Karnataka Health Promotion Trust, Bangalore, India ${ }^{4}$ Department of Community Health Sciences, University of Manitoba, Winnipeg, Canada ${ }^{5}$ Family Health International, Bangkok, Asia Pacific Regional Office, Bangkok, Thailand

\section{Correspondence to}

Ms Lakshmi Ramakrishnan, № 2 T2 Shivaji's Maniram Apt. Gandhi Mandapam Road, Kotturpuram, Chennai 600085 . India;

Iramakrishnan@fhiindia.org

Accepted 30 October 2009

\section{ABSTRACT}

Objective This paper evaluates Avahan programme's coverage of female sex workers (FSWs), focus on highrisk FSWs and intermediate outcomes.

Methods First round of cross-sectional survey data, Integrated Behavioral and Biological Assessments (IBBA), conducted in 22 districts, were aggregated into district categories: Solo, where Avahan was the sole service provider covering all FSWs and Major or Minor where Avahan was not the sole provider, but intended coverage was $>50 \%$ or $\leq 50 \%$ of FSWs respectively. Multivariate logistic regression was applied to compare exposure by district categories, vulnerability factors and intermediate outcomes associated with exposure.

Results Reported exposure, evaluated on basis of having received any of three core services, was higher in Solo (75\%) compared with Minor (66\%) districts. Logistic regression showed that FSWs in solo districts were more likely to be exposed (adjusted odds ratio $(A O R)=1.5$; $95 \% \mathrm{Cl} 1.20$ to 1.86 ) compared with FSWs in Minor districts. Multivariate analysis in Solo districts revealed that FSW with $\geq 15$ clients in the past week had a higher chance of being exposed to core services $(A O R=1.56$; $95 \% \mathrm{Cl} 1.03$ to 2.35). Exposure to the three services in Solo Avahan districts was significantly associated with correct knowledge on condom use $(\mathrm{AOR}=1.36 ; 95 \% \mathrm{Cl}$ 1.05 to 1.78), consistent condom use with occasional clients (AOR=3.17;95\% Cl 2.17 to 4.63) and regular clients $(\mathrm{AOR}=2.47 ; 95 \% \mathrm{Cl} 1.86$ to 3.28$)$ and STI treatment-seeking behaviour $(\mathrm{AOR}=3.00 ; 95 \% \mathrm{Cl} 1.94$ to 4.65).

Conclusions Higher coverage of FSWs was achieved in districts where Avahan was the only intervention compared with districts having multiple and longstanding non-Avahan programmes. Exposure in Solo districts was associated with intermediate outcomes; this need to be further evaluated in comparison with non Avahan areas and substantiated through data from next IBBA.

\section{INTRODUCTION}

India is now recognised as having diverse concentrated HIV epidemics, ${ }^{1-3}$ and as in other Asian countries, ${ }^{4}$ the dynamics of the epidemic are determined by the population sizes of sex workers and their clients. ${ }^{5} 6$ HIV prevalence among high-risk groups (HRGs) such as female sex workers (FSWs) range from $2 \%$ in Tamil Nadu to more than $30 \%$ in Karnataka and Maharashtra. ${ }^{7}$
In 2004, Avahan, the India AIDS Initiative funded by The Bill \& Melinda Gates Foundation started a \$250 million, 5-year HIV-prevention programme in the six most affected states for impacting HIV incidence among general population. 8 The programme was implemented in 83 districts, some where Avahan was the first or only programme (Solo) and others having concurrent non-Avahan programmes for FSWs. Avahan's objective was to rapidly scale-up and deliver a comprehensive package of proven HIV prevention services, which included peer-mediated education, STI treatment, condom promotion and distribution, and community mobilisation for greater ownership, among $\mathrm{HRGs}^{9}$ and to achieve coverage levels of over $80 \%$ in selected geographies. ${ }^{10}$ The aim was to address proximal and distal determinants of HIV risk, ${ }^{11}$ including consistent use of condoms, treatment of STIs, ${ }^{12}$ sex-work patterns and other environmental factors that make FSWs vulnerable to HIV and STIs, or that have shown to lower STI and HIV rates among HRGs. ${ }^{13-15}$

In India, targeted interventions for FSWs have been in operation since 1992, but few studies have been conducted to evaluate the coverage or service uptake and outcomes of programmes. Evaluation of the Sonagacchi project among FSWs in Kolkatta, using multiple rounds of cross-sectional surveys, indicated improved condom use and reduction in STI prevalence following intervention. ${ }^{15}$ A number of evaluation studies conducted outside India found effective FSW prevention programmes associated with improved knowledge, consistent use of condoms and better STI treatment seeking. ${ }^{16-21}$ Multiple rounds of Behavioural surveillance in China were analysed to establish a positive association between service coverage and behavioural outcomes such as improved consistent condom use among FSWs. ${ }^{22}$

This paper aims to present an analysis conducted as part of a larger evaluation of the Avahan programme among FSWs in India. Based on the Avahan programme's evaluation framework, ${ }^{8}$ the present analysis posed a set of questions to evaluate if the Avahan programme has achieved a higher coverage than other programmes, if there was sufficient focus on providing HIV prevention service to the most vulnerable subgroups of FSWs and achieving intermediate outcomes (consistent 
condom use with clients, knowledge of HIV transmission and STI treatment-seeking behaviours) using behavioural survey data collected from FSW in the 2 years following the start of intervention.

\section{METHODS}

As part of Avahan's evaluation design, ${ }^{8} 23$ two rounds of crosssectional surveys, termed Integrated Behavioural and Biological Assessment (IBBA), were planned, and the first was conducted between November 2005 and November 2007 and used for this paper. IBBA collected both behavioural information and biological specimens for HIV and STI testing among HRGs including FSWs.

\section{Settings and target population}

IBBA was conducted among FSWs in 25 of 83 Avahan districts, selected purposively based on two criteria: sociocultural region of the state and size of the FSW population. ${ }^{23}$ Eligible FSW survey participants were women aged 18 years or older, from either brothel-based or non-brothel-based (soliciting clients on streets or other non-brothel settings) settings who sold sex in exchange for cash at least once during the last 1 month.

\section{Sampling}

A representative sample of 400 FSW per district was selected through two-stage cluster sampling using probability proportional to size (PPS) method at the first stage and simple random sampling at the second stage. ${ }^{23}$ While conventional cluster sampling was used to sample venue-based FSWs from brothels, homes or lodges, the time location cluster sampling method was used to sample mobile FSWs from street-based settings. ${ }^{24} 25$ In some districts, separate samples for venue-based and non-venuebased (street-based) FSWs was taken due to the large size of these populations in these districts. ${ }^{23}$

\section{Ethical clearance and data collection}

Family Health International's (FHI) Protection of Human Subjects Committee and Ethical committees of implementing Indian Council of Medical Research (ICMR) institutes granted ethical clearances for IBBA. Local research agencies were provided training on survey procedures and conducted fieldwork. Face-toface interviews were administered in local language following informed consent, in private settings using precoded questionnaires. Data collection was initiated 2 to 27 months after the official launching of Avahan programme in 2005 and was completed in 2007. Further details of the complete IBBA survey and methodology can be referenced from previously published papers on IBBA round $\mathrm{I}^{23}$

\section{Considerations and analytical approach}

Key considerations for deciding on the analytical approach for analysis were: (1) IBBA districts included those where Avahan was the sole provider of services to FSWs and other districts where both Avahan and non-Avahan programmes, in different geographic areas, were providing services concurrently to $\mathrm{FSWs}^{8}$; (2) the Avahan programme's intended coverage (proportion of estimated total FSW population in the district targeted to covered by programme services) varied by districts and was decided prior to the start of Avahan programme $e^{26}$; (3) while IBBA attempted to collect reported exposure measure for a specific programme, it could not be used, as respondents could not always identify the specific service provider for a particular service. Given these considerations, the approach taken for analysis was to examine exposure by grouping districts into three categories on the basis of presence of non-Avahan interventions for FSWs and the Avahan programme's intended coverage of FSWs in the district and: (1) districts where Avahan is the only provider of HIV prevention services and intended coverage of FSWs was 100\% were termed 'Solo'; (2) districts having both Avahan and non-Avahan services for FSWs and where Avahan intended coverage greater than $50 \%$ of FSWs were termed 'Major'; and (3) districts having both Avahan and non-Avahan services for FSWs and where Avahan intended coverage was less than or equal to $50 \%$ of FSWs were termed 'Minor' districts. In Solo districts, detected exposure would primarily be that of the Avahan programme, whereas in Major and Minor district categories, detected exposure would be to any programme services, and Avahan-specific exposure could not be separated from exposure to other programmes. For lack of a purely non-Avahan control group, the Minor district category having the lowest intended Avahan coverage was considered to be a closest proxy for control group in the analysis.

\section{Measures}

Data on demographics, knowledge of HIV transmission, sexual behaviour, commercial sex work activity and uptake of services for HIV prevention and treatment of STIs were analysed. Based on the start date of any interventions in the district and IBBA survey dates, the duration of interventions at the time of data collection in IBBA districts was estimated (table 1).

\section{Exposure to services}

Exposure to service was defined as an aggregate variable based on proportion of FSW who reported having received any or all of the following core programme services in the past year (regardless of service provider): ${ }^{23}$ (1) contacted by a peer educator, (2) visited the programme clinic for STI services and (3) received condoms from peer or outreach workers. ${ }^{9} 27$ These are the three core services referred to in this paper.

\section{Most at risk FSWs}

In the analysis, the most at-risk FSWs were considered to be young FSWs (18-21 years old), FSWs new to sex-work (sold sex for $<1$ year), FSWs with large number of clients (client volume $\geq 15$ clients/week) and FSWs having no other source of income than sex work. These subgroups of FSWs were considered as most at risk, based on the conceptual and theoretical understanding that factors such as young age, shorter duration in sex work, high volume of clients and having no source of income are considered as distal determinants for HIV and STI risk. ${ }^{11}$

\section{Intermediate outcomes}

Intermediate outcomes considered were: (1) correct knowledge of HIV defined as providing the correct answer on HIV prevention through the use of condoms and HIV transmission through infected needles and rejecting two common misconceptions on transmission of HIV, by mosquito bite and by sharing food and clothing with infected persons; (2) consistent condom use with occasional clients; (3) consistent condom use with regular clients; and (4) treatment seeking from a trained healthcare provider for last STI symptom (refer to box 1).

\section{Data management and analysis}

Using variables commonly available across different district data sets, merged IBBA data were created for the analysis. Three districts were excluded in this analysis due to differences in sampling approach used or variations in the questionnaire 
Table 1 Proportion of exposure to services in Avahan districts

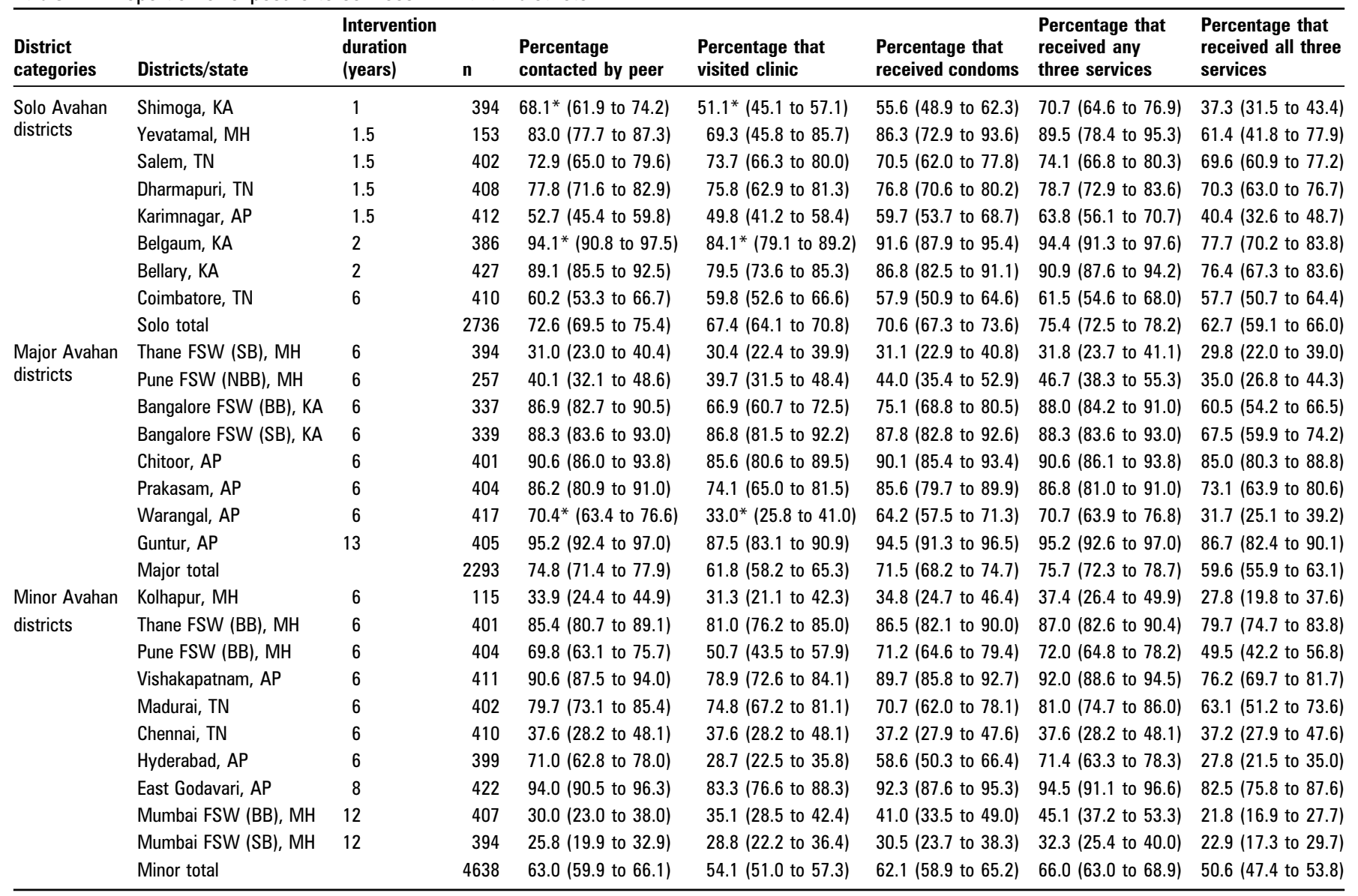

*Exposure to service in the last 6 months.

design. Weighted analysis was conducted using SPSS version 14.0 (SPSS, Chicago). Weights were calculated based on selection probabilities of clusters and of individual respondents. Further, specific weights were computed to provide estimates for different categories of Avahan districts (Solo, Major and Minor). ${ }^{25}$ Estimates were calculated along with $95 \%$ CI using the complex sample module of SPSS. ${ }^{28}$

\section{Comparisons in exposure}

Multiple logistic regressions using the forward method were used to calculate adjusted ORs (AOR) for comparing exposure in Solo and Major with respect to Minor districts. Separate models were constructed for each type of service and for all three types of services aggregated. In multiple regressions, the analyses were adjusted for age, typology of sex work, duration in sex work, literacy status and having other sources of income to account for differences in profile and sex-work characteristics across districts. Separate logistic regression models were applied to compare exposure to intervention in Solo with all other districts, Major and Minor, combined.

\section{Subgroup analysis in solo districts}

Analysis to assess associations between exposure (having received any of the three services) and focus on the most at-risk subgroups of FSWs as well as exposure and intermediate behavioural outcomes were restricted to solo Avahan districts. For the former, a multiple logistic regression was applied, and association was tested adjusting for typology of sex work (brothel or non-brothel based) and literacy among FSWs to account for district level variations. For the latter, four separate logistic regression models were constructed to assess associations between exposure and four intermediate outcomes indicated above. The model on treatment seeking for STIs was conducted as a subgroup analysis among FSWs who had experienced STI symptoms in the last year. Variables included in multivariate models were selected purposively, regardless of their level of association with the dependent or independent variables to account for differences in profile and sex work characteristics. These adjustment variables included: age, typology of sex work, duration in sex work, literacy and volume of clients. Age, duration in sex work and client volume were taken as continuous variables in the models.

Each of the above logistic regression models was analysed using the complex samples module in SPSS version 14.0, where the null hypothesis was tested based on a $p$ value of $<0.05$ and $95 \%$ CI not including 1.

\section{RESULTS}

Districts retained for analysis included eight in Solo, eight in Major and 10 in Minor district categories, and represented a total of 9667 FSW participants (28\% in Solo, $24 \%$ in Major and $48 \%$ in Minor). The response rate for participation in IBBA ranged between $44.1 \%$ and $89.9 \%{ }^{7}$

\section{Profile of FSWs}

Comparison of profile characteristics by district category revealed significant differences. The mean age in Solo was 32 years, compared with 30 in both Major and Minor category. There was a higher proportion of FSWs aged 18 to 21 years in 
Minor (9\%) and Major (13\%) districts compared with Solo districts $(7 \%)\left(\chi^{2} \mathrm{p}\right.$ value $\left.=0.000\right)$.

Home and street-based FSWs comprised the majority in Solo (23\% and $63 \%$ respectively) and Major (30\% and 61\%), whereas brothel and street-based FSWs were the majority in Minor districts (35\% and 55\%). A higher proportion in Solo were literate $(35 \%)$ compared with Major $(27 \%)$ or Minor districts $(30 \%)\left(\chi^{2}\right.$ p value $\left.=0.000\right)$. In Minor category, a higher proportion of FSWs reported no other income source (66\%) compared with Solo $(60 \%)$ and Major $(54 \%)$ districts $\left(\chi^{2}\right.$ p value $\left.=0.000\right)$.

\section{Evaluated reported exposure in Avahan districts}

Exposure to intervention, having received any of the three core HIV prevention services, was highest in Solo (75\%) and Major $(76 \%)$ than in Minor (66\%) districts (table 1). Contact with peer educators was the service most frequently received by FSW in all three intended coverage areas, whereas uptake of STI clinic services was the least reported. Exposure to all of the three core services was also highest in Solo (62.7\%) followed by Major (59.6\%) and Minor (50.6\%) districts. The mean duration of interventions in Solo was 2.1 years, compared with 6.8 years in Major and 7.4 years in Minor districts.

In multivariate analysis FSW in Solo (AOR=1.49; 95\% CI 1.20 to 1.86 ) and Major ( $\mathrm{AOR}=1.56$; $95 \% \mathrm{CI} 1.25$ to 1.96 ) were more likely to have received any of the three core services compared with FSWs in Minor districts (table 2). Compared with Minor districts, FSWs in Solo districts were more likely to have been contacted by peer educators (AOR: 1.39; 95\% CI 1.24 to 1.55), received condoms (AOR: 1.55 ; $95 \%$ CI 1.30 to 1.61 ) and visited programme STI clinics (AOR=1.65; 95\% CI 1.49 to 1.83) (table 2). Similarly, FSWs in Major districts were more likely to have received any of the three core services compared with FSWs in Minor Avahan districts, Logistic regression comparing aggregate exposure measure in Solo districts versus all other districts combined showed that reported exposure in Solo districts was significantly higher $(\mathrm{OR}=1.28 ; 1.06$ to 1.31$)$ than reported exposure in other districts (data not in table).

\section{Exposure among most at risk FSWs in Solo districts}

Multivariate analysis restricted to Solo districts revealed that FSWs who were 18-21 years were less likely to have received any of the three core services ( $A O R=0.46 ; 95 \%$ CI 0.29 to 0.74 ) compared with those aged over 30 years; and similarly those in sex work for $<1$ year were less likely have received the three core services ( $\mathrm{AOR}=0.70 ; 95 \% \mathrm{CI} 0.459$ to 1.071 ) compared with $6+$ years in sex work, whereas FSWs reporting a high client volume of 15 or more clients in the past week (compared with FSW with fewer than 15 clients in the past week) were more likely to have received any of the three core services (table 3 ). No difference in exposure was observed by FSW's source of income.

\section{Achievement of Intermediate outcomes in Solo districts}

FSWs exposed to any of three core services in Solo Avahan districts had accurate knowledge (43\%) and were using condoms consistently with occasional $(75 \%)$ or regular $(72 \%)$ clients and seeking treatment for STIs (90\%) (table 4). The logistic regression models revealed that each of the intermediate outcomes was significantly associated with having received any of three core services in Solo Avahan districts in the past year: correct knowledge on HIV (AOR: 1.36; 95\% CI 1.05 to 1.78), consistent condom use with occasional clients (AOR: 3.17; 95\% CI 2.17 to 4.63); consistent condom use with regular clients (AOR: 2.46; $95 \%$ CI 1.87 to 3.82 ) and seeking treatment for STIs from a trained healthcare practitioner in the past year (AOR: 3.00; 95\% CI 1.94 to 4.65 ) (table 4)

\section{DISCUSSION}

Using data from IBBA, the current paper has attempted to evaluate the exposure and intermediate outcomes in a subset of Avahan programme intervention districts across India. A key finding from the present analysis was that FSWs in Solo and Major Avahan districts were more likely to have been reached by HIV prevention programmes than FSWs in Minor districts with longer-standing non-Avahan interventions. While in the majority of Solo districts, Avahan was the first provider of services for FSWs, three districts (Bellary, Belgaum and Coimbatore) had some previous interventions for FSWs, though the coverage of these interventions is not known. Apart from these three districts, the high coverage $(63.8 \%$ to $89.5 \%)$ in the other districts, where Avahan was the first and solo provider of services for FSWs, was achieved within 2 years of initiation of intervention.

The National AIDS Control Organization (NACO) in India estimates that at the end of the second phase on National AIDS

Table 2 Multivariate analysis of exposure by Avahan district categories

\begin{tabular}{|c|c|c|c|}
\hline & $(\%)(\mathbf{n})^{*}$ & Crude OR $(95 \% \mathrm{CI})$ & Adjusted + OR (95\% CI) \\
\hline \multicolumn{4}{|c|}{ a. Exposure to any of three core services in last year (no and yes) } \\
\hline Minor Avahan & $66.0(4638)$ & 1.00 & 1.00 \\
\hline Major Avahan & $75.7(2293)$ & $1.59 * *(1.28$ to 1.99$)$ & $1.56^{* *}(1.25$ to 1.96$)$ \\
\hline Solo Avahan & $75.4(2736)$ & $1.58^{* *}(1.29$ to 1.94$)$ & $1.49^{* *}(1.20$ to 1.86$)$ \\
\hline \multicolumn{4}{|c|}{ b. Contacted by peer in last year(no and yes) } \\
\hline Minor Avahan & (4638) & 1.00 & 1.00 \\
\hline Major Avahan & $74.8(2293)$ & $1.74^{* *}(1.40$ to 2.15$)$ & $1.63^{* *}(1.45$ to 1.83$)$ \\
\hline Solo Avahan & $72.6(2736)$ & $1.55^{* *}(1.27$ to 1.88$)$ & $1.38^{* *}(1.24$ to 1.55$)$ \\
\hline \multicolumn{4}{|c|}{ c. Dependent variable: visited clinic in last year (no and yes) } \\
\hline Minor Avahan & (4638) & 1.00 & 1.00 \\
\hline Major Avahan & $61.8(2293)$ & $1.37^{* *}(1.28$ to 1.66$)$ & $1.32^{* *}(1.18$ to 1.47$)$ \\
\hline Solo Avahan & $67.4(2736)$ & $1.75^{* *}(1.44$ to 2.13$)$ & $1.65^{* *}(1.48$ to 1.83$)$ \\
\hline \multicolumn{4}{|c|}{ d. Dependent variable: received condom (no and yes) } \\
\hline Minor Avahan & (4638) & 1.00 & 1.00 \\
\hline Major Avahan & $61.8(2293)$ & $1.53^{* *}(1.24$ to 1.89$)$ & $1.55^{* *}(1.38$ to 1.74$)$ \\
\hline Solo Avahan & $67.4(2736)$ & $1.46^{* *}(1.19$ to 1.79$)$ & $1.45^{* *}(1.30$ to 1.61$)$ \\
\hline
\end{tabular}

\footnotetext{
*\%, proportion of female sex workers exposed; $n$, total number of female sex workers in the respective categories.

†Adjusted for age, typology, duration in sex work, literacy and income source.

${ }^{* *} \mathrm{p}<0.01$.
} 
Table 3 Multivariate analysis of exposure by female sex worker subgroups in Solo Avahan districts

\begin{tabular}{|c|c|c|c|}
\hline Particulars & $(\%)(n) \dagger$ & Crude OR (95\% CI) & Adjusted $\neq$ OR $(95 \% \mathrm{CI})$ \\
\hline \multicolumn{4}{|c|}{ Current age (years) } \\
\hline $30+$ & $76.0(1765)$ & 1.00 & 1.00 \\
\hline $18-21$ & $63.3(215)$ & $0.54^{* *}$ & $0.46^{* *}(0.29$ to 0.74$)$ \\
\hline $22-25$ & $72.3(349)$ & 0.83 & $0.79(0.50$ to 1.24$)$ \\
\hline $26-29$ & $82.0(490)$ & $1.44^{*}$ & $1.55^{* *}(1.13$ to 2.12$)$ \\
\hline \multicolumn{4}{|c|}{ Have another source of income } \\
\hline Yes & $75.6(1759)$ & 1.00 & 1.00 \\
\hline No & $75.6(1176)$ & $1.00(0.76$ to 1.33$)$ & $0.97(0.74$ to 1.27$)$ \\
\hline \multicolumn{4}{|c|}{ Duration of sex work (years) } \\
\hline $6+$ & $80.6(1100)$ & 1.00 & 1.00 \\
\hline $2-5$ & $72.7(1328)$ & $0.60^{* *}(0.42$ to 0.87$)$ & $0.72^{* *}(0.52$ to 1.00$)$ \\
\hline$\leq 1$ & $71.5(522)$ & $0.64^{* *}(0.48$ to 0.85$)$ & $0.70^{*}(0.46$ to 1.07$)$ \\
\hline \multicolumn{4}{|c|}{ Client volume (clients/week) } \\
\hline$\leq 14$ & $73.9(2466)$ & 1.00 & 1.00 \\
\hline$\geq 15$ & $83.0(471)$ & $1.73^{* *}(1.14$ to 2.63$)$ & $1.56^{* *}(1.03$ to 2.35$)$ \\
\hline
\end{tabular}

Control Program (NACP) II, more than half the targeted HIV prevention interventions were in the high-prevalence states of Andhra Pradesh, Karnataka, Maharashtra, Manipur, Nagaland and Tamil Nadu with an overall estimated coverage of $45 \%$ to $50 \%$ of all HRGs. ${ }^{29}$ The National Behavioral Surveillance conducted among FSWs in 25 Indian states in 2006, using a different sampling approach, reported that $56 \%$ of FSWs at the national level had reported receiving information on HIV and STIs in the previous 1 year from any HIV-prevention interventions. $^{30}$ Though these data are not directly comparable with IBBA, they suggest that the coverage by the Avahan programme, as indicated in the Solo districts, was greater than the reported coverage at the national level, from 25 states, the majority of which were having non-Avahan programmes.

A main strategy of the Avahan programme was to address the proximate and distal determinants of HIV risk ${ }^{9} 12$ among FSWs. The Sonagacchi project for FSWs, a successful model of HIV prevention from India, focused on vulnerable subgroups of FSWs: younger FSWs, FSWs new to sex work and FSWs with a higher volume of clients. ${ }^{31}$ In Solo districts, intervention coverage was higher among FSWs with a higher client volume but lower among the other at-risk FSWs, those younger in age and those who recently came into sex work. These results suggest that Avahan programme needs to develop further strategies to identify and provide HIV prevention services to FSWs who are younger and have recently been initiated into sex work.

The analysis indicates that intermediate outcomes such as knowledge, condom use and treatment-seeking behaviour were significantly associated with exposure to intervention in Solo Avahan districts. These outcomes were achieved in less than 2 years of initiation of Avahan interventions. FSWs exposed to HIV prevention programmes in these districts were more likely to have accurate knowledge of HIV. Using a consistent definition, correct knowledge among FSWs exposed to programmes was higher in Solo districts (43\%) compared with that reported by the National BSS (2006) among all FSWs (38\%) in India. ${ }^{30}$

Several other studies in India have shown improved condom use after HIV prevention interventions among FSWs. ${ }^{15} 3233$ Our analysis in Solo districts also revealed that the majority of FSWs were consistently using condoms, $75 \%$ with occasional clients and $72 \%$

Table 4 Multivariate models (4) of intermediate outcomes by exposure in Solo Avahan districts

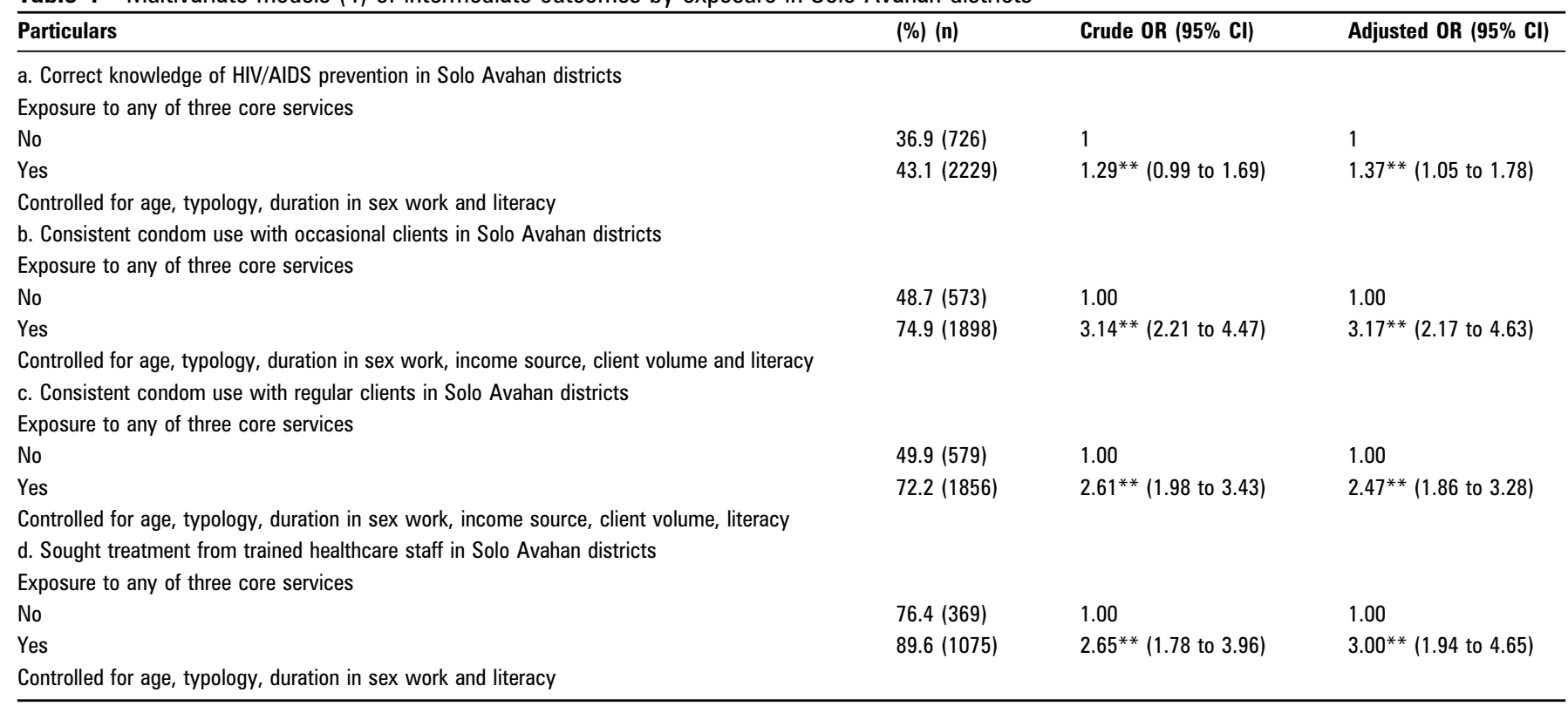


with regular clients, and this was significantly associated with exposure to package of HIV prevention services in these districts.

Another component of the Avahan programme strategy included the STI service package (treatment of symptomatic and asymptomatic FSWs as well as monthly screening), which was shown to be effective for reducing the STI rate among FSWs. ${ }^{12} 34$ In Solo districts, more sex workers exposed to intervention had sought treatment for STIs from trained practitioners than nonexposed FSWs, suggesting that the peer education contributed to improved health-seeking behaviours among FSWs.

The main limitation of the current analysis is the lack of a truly non-Avahan control group for comparison of intermediate outcomes achieved by Avahan with other non-Avahan programmes. Due to poor recall and difficulty of FSWs to distinguish between service providers, it was difficult to distinguish between exposure to Avahan and non-Avahan interventions using IBBA data. In districts with longstanding interventions, FSWs were able to recall the services provided but not the programme name. While the Avahan programme in each state had a specific brand name, this was not the case for many other non-Avahan programmes. Further, interventions across districts were in different stages of implementation at the time of IBBA, which meant that their extent of branding varied, possibly higher in some districts than in others. These issues made it difficult to distinguish between Avahan and non-Avahan exposure in mixed intervention districts, while in the Solo districts Avahan was the only programme providing peer education, condom promotion and STI clinical services to FSWs. The approach of categorising districts into the three categories therefore provided a way for comparing coverage between district categories with Minor as the closest proxy for nonAvahan group. Other limitations that complicate the comparison and interpretation of coverage are the lack of information on the levels of existing coverage by HIV prevention services in the Solo, Major and Minor districts prior to the Avahan programme and the lack of randomised of districts on the basis of intended Avahan coverage.

The limitations of a cross-sectional survey apply to this current analysis, in that the direction of relationship between exposure to interventions and intermediate outcome cannot be interpreted. ${ }^{35}$ While it is not possible to rule out that the intermediate outcomes seen here could be independent of the interventions, without having an appropriate control group, the magnitude of coverage and outcomes from the present analysis however make this possibility less likely. More conclusive associations or trends can best be established based on successive rounds of IBBA data.

\section{Key messages}

- Data on coverage by HIV prevention programmes for female sex workers are presented from 22 districts across highprevalence Indian states.

- Using cross-sectional survey data aggregated from 22 districts, this paper presents an evaluation of coverage and associated outcomes.

- The Avahan programme in India has achieved a high coverage of female sex workers across intervention areas.

- Outcomes such as condom use and STI treatment-seeking behaviour are associated with exposure to services in districts having only the Avahan programme, a large-scale HIVprevention programme in India.
Though categorising districts and aggregating the data for analysis gives statistical power and informs the programme at pan-Avahan level, it does not account for district-level variations in epidemic patterns, characteristics of FSW, and the duration and nature of HIV-prevention interventions over time that would affect coverage and intermediate outcomes. Therefore, further district or regional analyses would help to tease out the district/regional level differences that may get masked in an aggregate analysis. Yet the current analysis presented in this paper provides a potential approach to evaluate coverage and measurement of intermediate outcomes among FSWs over time, when data from future rounds of IBBA are available.

\section{CONCLUSION}

The current analysis indicates that programmes in districts where Avahan was a solo programme had achieved a high scale of coverage in a shorter time than with other categories of districts with longer history of interventions. Further, exposure to these programmes was associated with accurate knowledge, consistent condom use with clients and positive health-seeking behaviour among FSWs. Further rounds of IBBA will allow constructing trends in intermediate outcomes in Avahan districts compared with other districts.

Acknowledgements The authors would like to thank all the principal investigators and project staff of the ICMR Institutes, National AIDS Research Institute, National Institute of Epidemiology and National Institute of Nutrition, who implemented IBBA in their State. We would also like to extend our thanks to the professional research agencies, TNS India and AC Nielson ORG-MARG, and their staff who carried out the field data collection for the study. Thanks also to the Avahan State Implementing partners and NGOs and the FSW community in each state for their cooperation and participation

Contributors LR was responsible for concept development, analysis, the writing and finalisation of manuscript; $A G$ and PG were responsible for concept design, data management and analysis and finalisation of manuscript; SK was involved in data collection and data management; RA was responsible for guiding the planning and execution of the study and contributed to design, analysis and the development and finalisation of manuscript; MM coordinated the study and contributed to analysis; BM provided data and technical assistance to analysis, GM provided technical assistance for analysis, developing and editing manuscript; BG contributed to development and finalisation of manuscript; and RP was the principal investigator for the study.

Funding This research and the Avahan programme were funded by The Bill \& Melinda Gates Foundation. The funding agency did not have any role in collection, analysis or interpretation of data; in the writing of the report; or in the decision to submit the paper for publication

The views expressed herein are those of the authors and do not necessarily reflect the official policy or position of the Bill \& Melinda Gates Foundation.

\section{Competing interests None.}

Ethics approval Ethics approval was provided by the India and USA

Provenance and peer review Not commissioned; externally peer reviewed.

\section{REFERENCES}

1. Claeson M, Alexander A. Tackling HIV in India: evidence-based priority setting and programming. Health Aff 2008;27:1091-101.

2. Chandrasekaran P, Dallabetta G, Loo V, et al. Containing HIV/AIDS in India: the unfinished agenda. Lancet Infect Dis 2006;6:508-21.

3. Moses S, Blanchard JF, Kang H, et al. AIDS in South Asia: understanding and responding to a heterogeneous epidemic. The World Bank, Washington DC: 2006.

4. Dore GJ, Kaldor JM, Ungchusak K, et al. Epidemiology of HIV and AIDS in the AsiaPacific region: an epidemiological overview. AIDS 1998;12(Suppl B):S1-10.

5. Nagelkerke NJD, Jha P, Vlas SJD, et al. Modeling HIV/AIDS epidemics in Botswana and India: impact of interventions to prevent transmission. BullWHO 2002;80:89-96.

6. Ruxrungtham K, Brown T. HIV/AIDS in Asia. Lancet 2004;364:69-82.

7. Banakkoppa RM, Moses S, Washington R, et al. Determinants of HIV prevalence among female sex workers in four south Indian states: analysis of cross-sectional surveys in twenty-three districts. AIDS 2008;22(Suppl 5):S35-44.

8. Chandrasekaran P, Dallabetta G, Loo V, et al. Evaluation design for measuring the impact of the first phase of a large scale HIV prevention effort in India: Avahan, the India AIDS initiative. AIDS 2008;22(Suppl 5):S1-15.

9. Avahan, the India AIDS Initiative-the Business of HIV prevention at Scale. The Bill 8 Melinda Gates Foundation, New Delhi, India. 2008. http://www.gatesfoundation.org/ avahan/Documents/Avahan_HIVPrevention.pdf (accessed Jan 2009). 
10. Coates TJ, Richter L, Caceres C. Behavioral strategies to reduce HIV transmission: how to make them work better. Lancet Series. Published Online First 6 Aug 2008. doi: 10.1016/So140-6736(08)60886-7.

11. Boerma JR, Weir SS. Integrating demographic and epidemiological approaches to research on HIV/AIDS: The proximate-determinants framework. J Inf Dis 2005:191 (Suppl 1):S61-7.

12. Steen R, Dallabetta G. Sexually transmitted infection control with sex workers regular screening and presumptive treatment augment efforts to reduce risk and vulnerability. Reprod Health Matters 2003:11:74-90.

13. Ghys PD, Diallo MO, Ettiègne-Traoré V, et al. Increase in condom use and decline in HIV and sexually transmitted diseases among female sex workers in Abidjan, Cote d'lvoire, 1991-1998. AIDS 2002;16:251-8.

14. Alary $\mathbf{M}$, Lowndes CM, Gnintounbgè CAB, et al. Decline in the prevalence of HIV and sexually transmitted diseases among female sex workers in Cotonou, Benin, 1993-1998. AIDS 2002;16:463-70.

15. Jana S, Bandyopadhyay N, Mukherjee S, et al. STD/HIV intervention with sex workers in West Bengal, India. AIDS 1998:12(Suppl B):S101-8.

16. Asamoah-Adu A, Weir S, Papoe M, et al. Evaluation of a targeted AIDS prevention intervention to increase condom use among prostitutes in Ghana. AIDS 1994:8:239-46.

17. Rou K, Wu Z, Sullivan SG, et al. A five-city trial of a behavioral intervention to reduce sexually transmitted disease/HIV risk among sex workers in China. AIDS 2007:21 (Suppl 8):S95-101.

18. Wu Z, Rou K, Jia M, et al. The first community-based sexually transmitted disease/ HIV intervention trial for female sex workers in China. AIDS 2007:21(Suppl 8): S89-94.

19. Van Griensven GJP, Limanonda B, Ngaokeow S, et al. Evaluation of a targeted HIV prevention programme among female commercial sex workers in south of Thailand. Sex Transm Infect 1998:74:54-8.

20. Ford K, Wirawan DN, Suastina W, et al. Evaluation of a peer education programme for female sex workers in Bali, Indonesia. Int J STD AIDS 2000:11:731-3.

21. Walden MV, Mwangulube K, Nakhumula-Nkhoma P. Measuring the impact of a behaviour change intervention for commercial sex workers and their potential clients in Malawi. Health Educ Res 1999;4:545-54.

22. Lau JTF, Choi KC, Tsui HY, et al. Changes in HIV-related behaviours over time and associations with rates of HIV-related services coverage among female sex workers in Sichuan, China. Sex Transm Inf 2008;84:212-16.
23. Saidel T, Adhikary R, Mainkar M, et al. Baseline integrated behavioral and biological assessment among most at-risk populations in six high-prevalence states of India: design and implementation challenges. AIDS 2008:22(Suppl 5):S17-34.

24. Kalton G. Sampling: How's of mobile human populations. Surv Methodol 1991;17:183-194

25. Family Health International. Repeated behavioral surveillance surveys: guidelines for repeated behavioral surveys in populations at risk of HIV. New Delhi, India: Family Health International, 2000

26. Verma R, Shekhar A, Khobragade S, et al. Scale-up and coverage of Avahan: a largescale HIV-prevention programme among female sex workers and men who have sex with men in four Indian states. Sex Transm Infect 2010:86(Suppl 1):i76-82.

27. Blanchard JF, Bhattarcharjee P, Kumaran S, et al. Concepts and strategies for scaling up focused prevention for sex workers in India. Sex Transm Infect 2008;84 (Suppl 2):ii19-23.

28. Fleiss JL, Levin B, Cho Paik M. Statistical methods for rates and proportions, 3rd edn New Jersey, USA: Wiley Interscience 2003:308-31)18.

29. National AIDS Control Organization. India. NACP III Project Implementation Plan. 2006.

30. National AIDS Control Organization (NACO) the Ministry of Health and Family Welfare and Center for Social Research India. National Behavioral Surveillance Survey (BSS), 2006, Female sex workers and their clients. New Delhi: National AIDS Control Organization (NACO), the Ministry of Health and Family Welfare and Center for Social Research, 2007. http://www.nacoonline.org/Quick Links/Publication/ ME and Research Surveillance/Reports and Surveys/National BSS 20062/ (accesse-d Nov 2008)

31. Gangopadhyay DN, Chanda M, Sarkar K, et al. Evaluation of sexually transmitted diseases/human immunodeficiency virus intervention programs for sex workers in Calcutta, India. Sex Transm Diss 2005;32:680-4.

32. Dandona $\mathbf{R}$, Dnadona L, Gutierrez JP, et al. High risk of HIV in non-brothel based female sex workers in India. BMC Public Health 2005;5:87-96.

33. Bhave G, Lindan CP, Hudes ES, et al. Impact of an intervention on HIV, sexually transmitted diseases, and condom use among sex workers in Bombay, India. AIDS 1995;9(Suppl 1):S21-30.

34. Mogasale SR, Wi T, Singh AK, et al. Pursuing scales and quality in STI interventions with sex workers: initial results from Avahan India AIDS Initiative. Sex Transm Inf 2006;82:381-5.

35. Zaba B, Slaymaker E, Urassa M, et al. The role of behavioral data in HIV surveillance. AIDS 2005:19(Suppl 2):S39-52. 